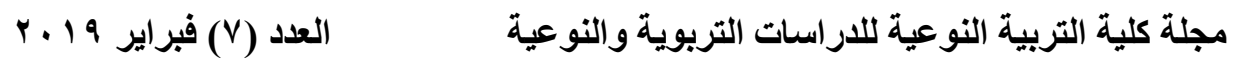

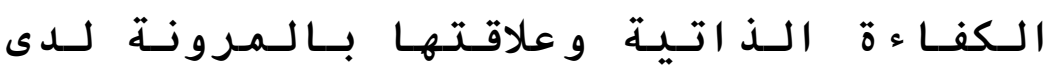

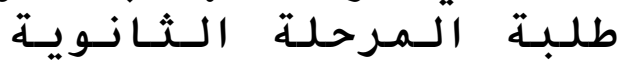

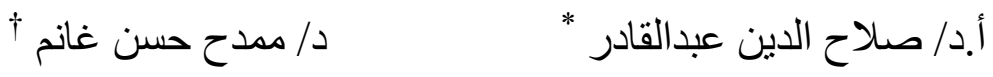

\title{
مقدمة تعثرة
}

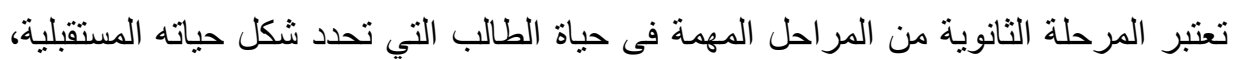

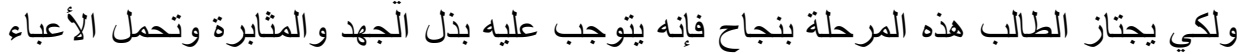

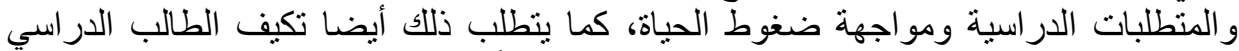

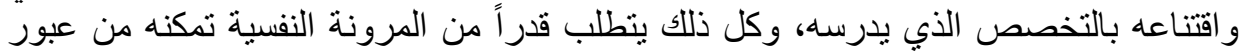

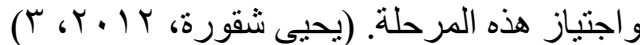

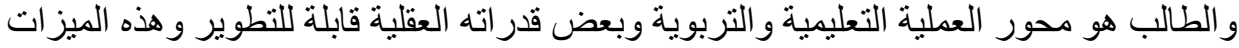

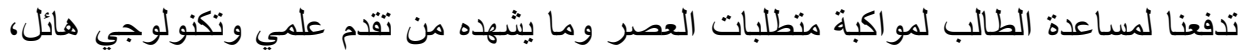

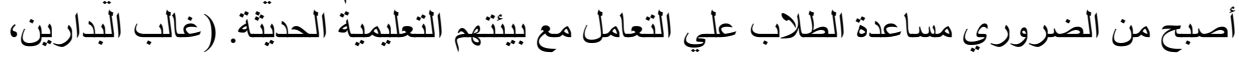

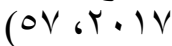

وتعد المرونة أحد العمليات التي تمكن الإنسان من التكيف مع مو اقف الحئ الحياة المختلفة ومنها البيئة

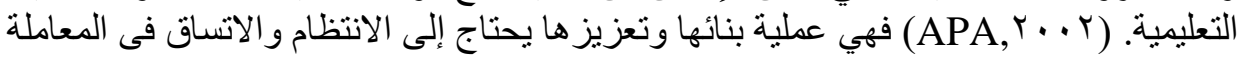

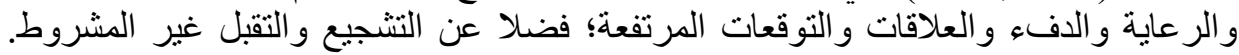

(Wang, m, 199v, 7r)

حيث لاغني عن المرونة فى التكيف مع المستجدات و المعلومات الجديدة التي يواجهها الأفراد

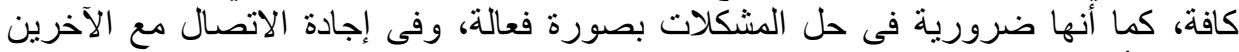

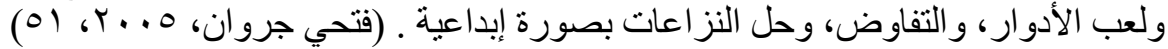

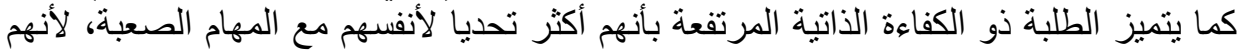

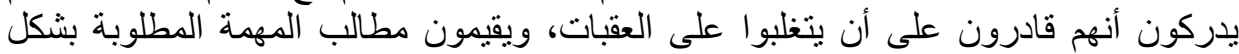

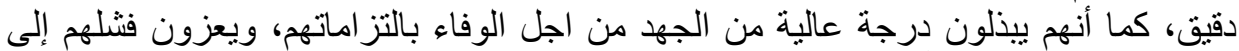

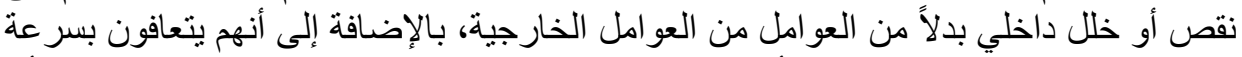

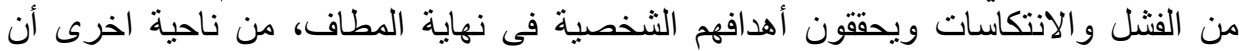

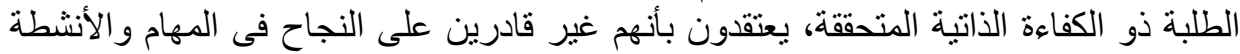

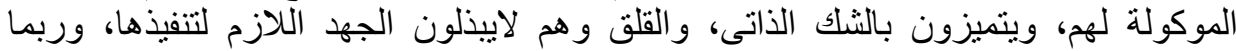

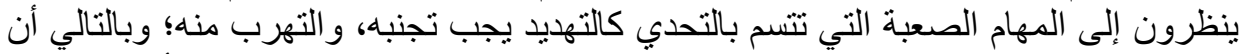

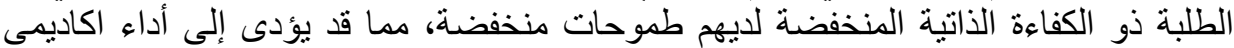

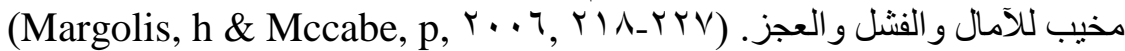

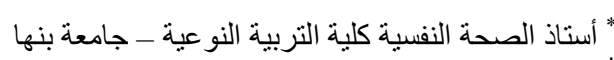

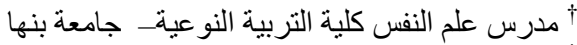

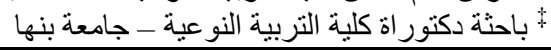


تتحدد مشكلة البحث فى السؤال الرئيسي النالي هل توجد علاقة ارتباطيه موجبة دالة إحصائيا بين درجات طلاب المجمو علة لمقياس المرونة ومقياس الكفاءة الذاتيةج أهداف الاراسة

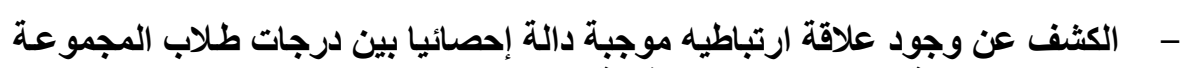
لمقياس المرونة ومقياس الكفاءة الذاتية.

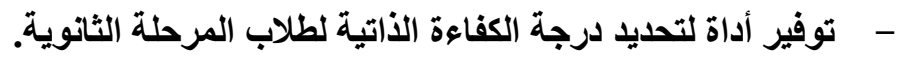
- - توفير أداة لتحديد درجة المرونة لطلاب المرحلة الثانوية.

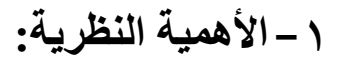
- يعتبر هذا البحث من الأبحاث التي تنتقدها المؤسسات التعليمية، وذلك فى حدود اطلاع الباحثة.

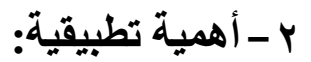

- بناء على نتائج البحث المتوقعة يمكن إعداد وتطبيق برامج إرشادية تهدف إلى تنمية

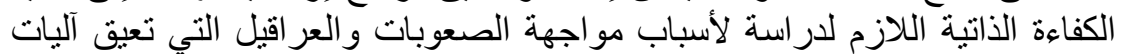

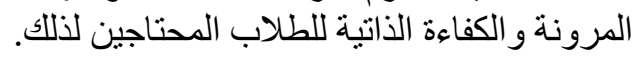

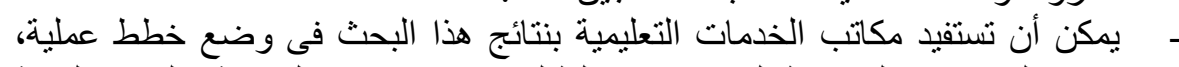
تؤدى إلى خفض الضغوط التي يو اجهها الطالب ورفع مستوى المرونة و الكفاءة الذاتية

الحدود الموضوعية: سوف يقتصر البحث على معرفة العلاقة بين الكفاءة الذاتية و الدرونة لدى طلبة المرحلة الثانوية.

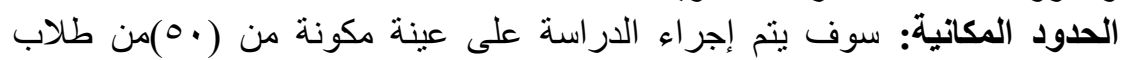

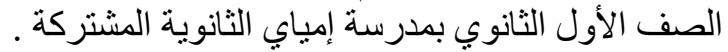

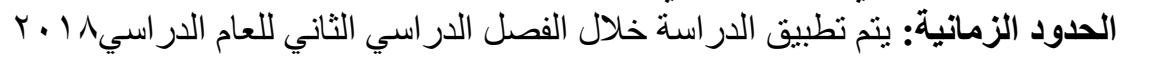

$$
\text { مصطلحات الدراسة }
$$

ه الكفاءة الذاتية وتعرف على أنها " معتقدات شخصية عند اداء الفرد لمهار اته الذاتية،

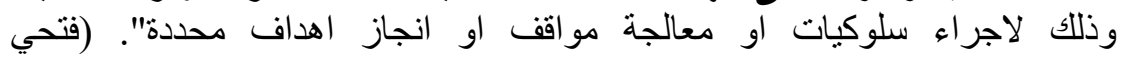

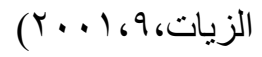
المرونة وتعرف على أنها " قدرة الفرد على الحفاظ على توازنها، وعلى تو افقه الجيد

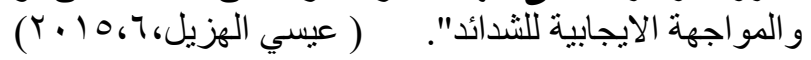




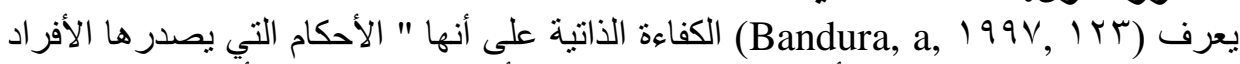

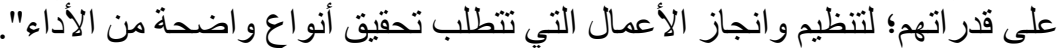

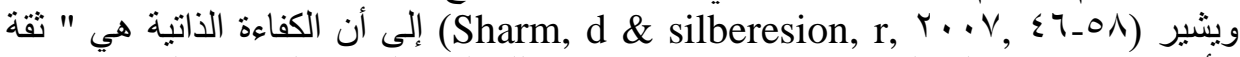

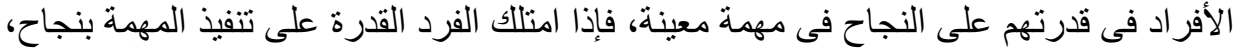

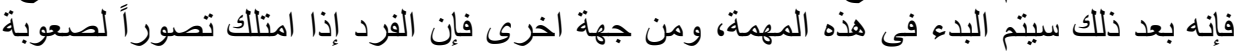

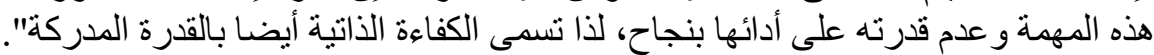

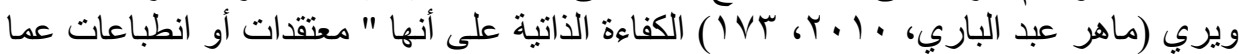

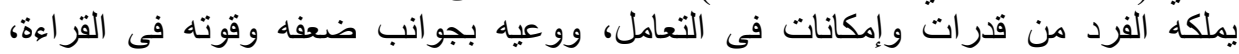

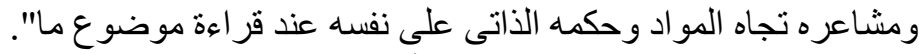

الخصائص المميزة للكفاعة الذاتية التية التئية

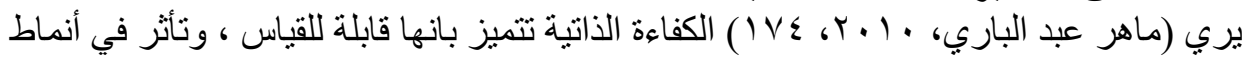

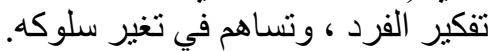

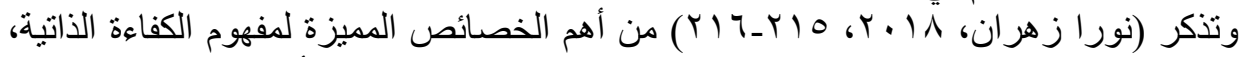

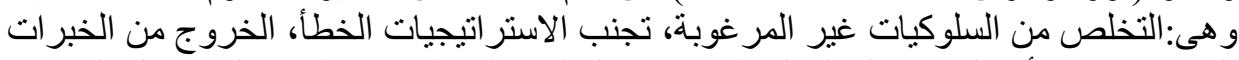

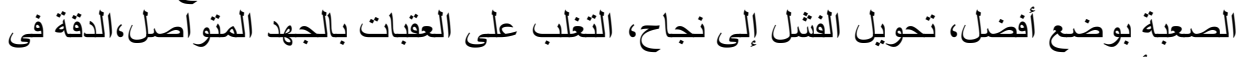

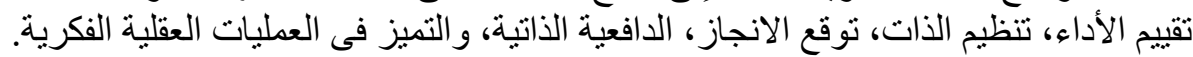

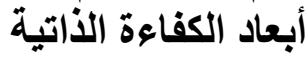

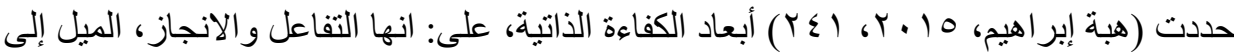
التعلم، المثابرة، و إدر الك الذات الذات

مصادر الكفاءة الأتية وائة

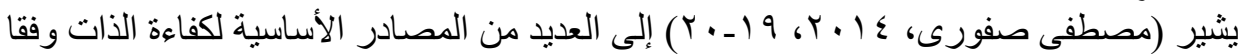

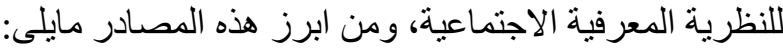

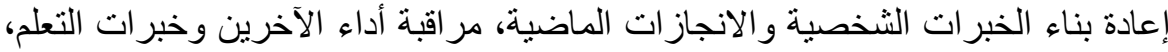

الإقناع اللفظى، و الحالة الفسيولوجية و العاطفة.

دراسات تناولت الكفاءة الذاتية والمرحلة الثانوية

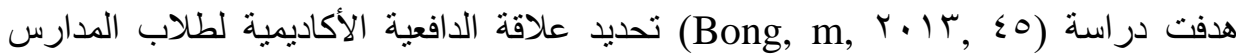

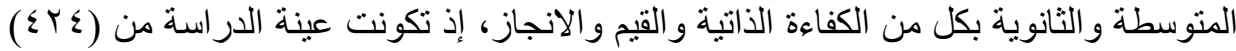

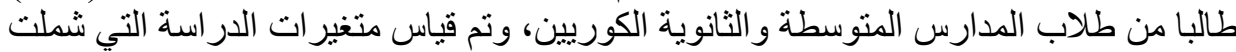

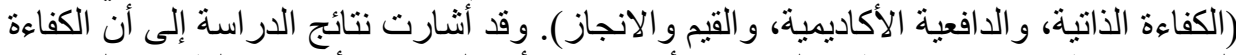

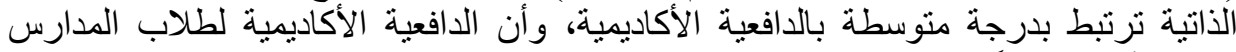

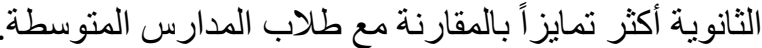

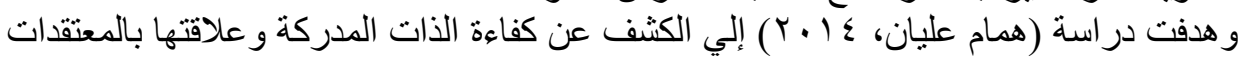

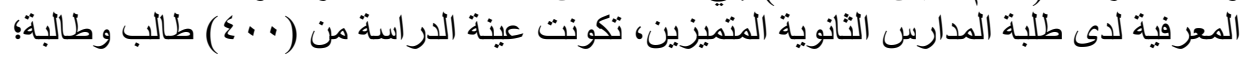


ولتحقيق أهداف الدراسة قام الباحث ببناء مقياس الكفاءة الذاتية، وأثنارت النتائج إلى أن طلبة النقاء

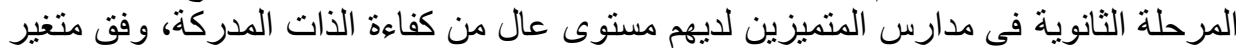
الجنس (ذكور وإناث) لصالح الذكور، وأن كفاءة الذات المدركة للأكور ومعتقداتهم المعرفية الذانية

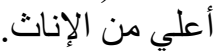

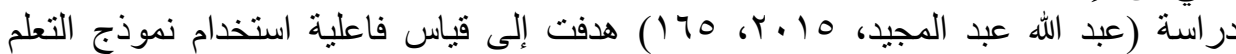

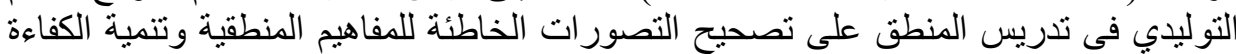

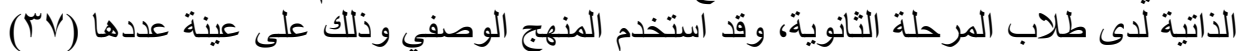

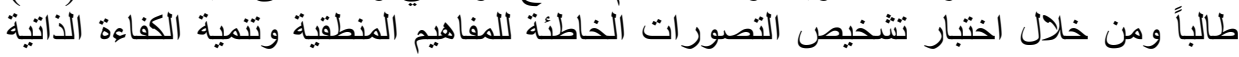

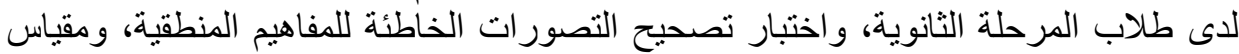

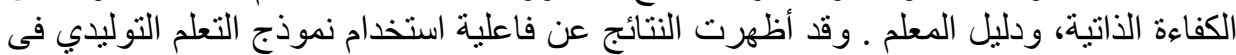
تدريس المنطق على تصحيح التصورات الخاطئة للمفاهيم المنطقية وتنمية الكفاءة الذية الذاتية لدى لئ طلاب المرحلة الثانوية.

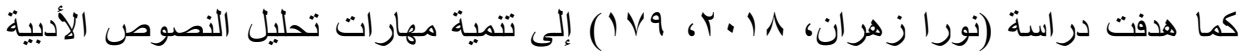

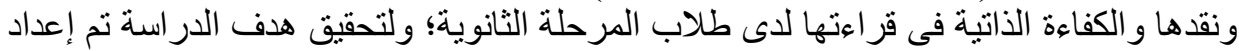

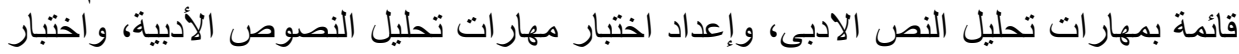

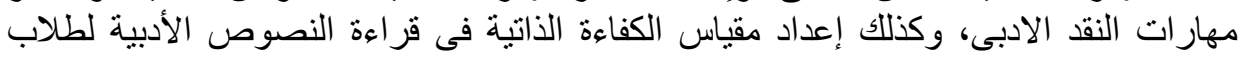

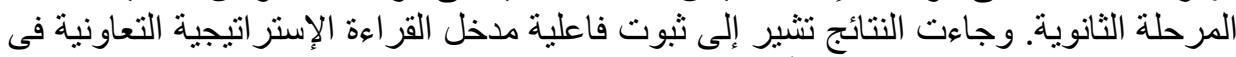

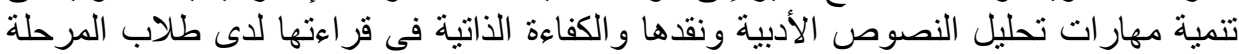
الثانوية.

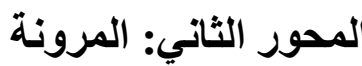

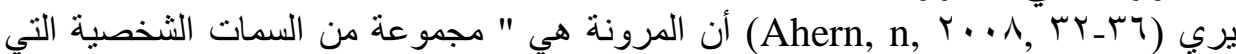

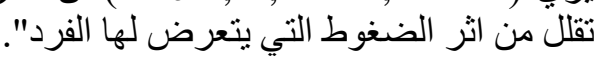

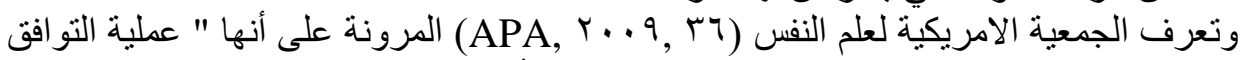

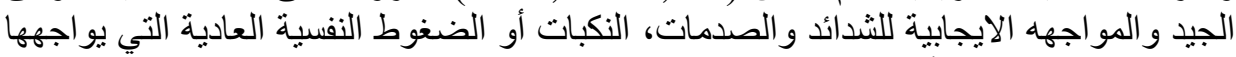

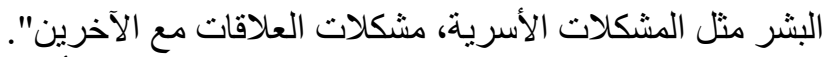

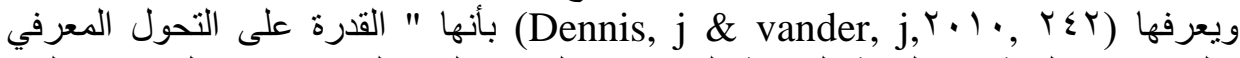
و التكيف مع المؤثرات البيئية الصعبة المتغيرة، و القدرة على توليد و إنتاج بدائل متعددة لهذه

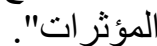

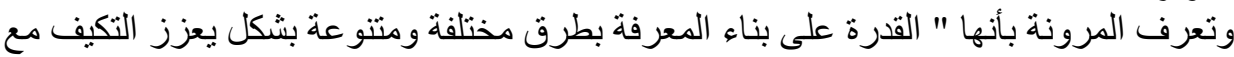

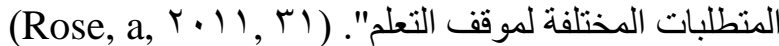

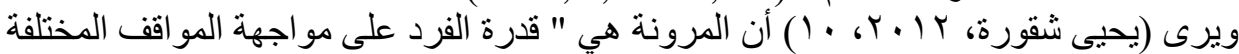

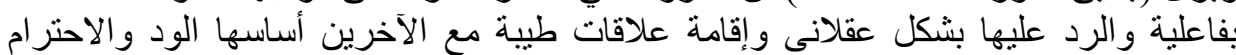

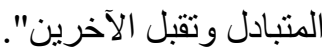

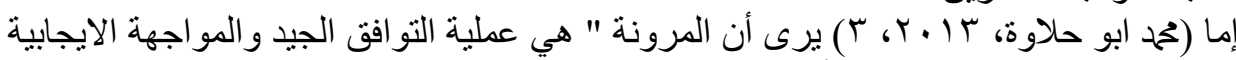
للثدائد، و الصدمات، و النكبات، أو الضغوط النوان النفسية العادية التي يو اجهها البشر ". 


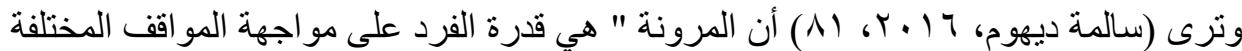
فى حدتها وشدتها، وذللك بالمناعة الايمانية المكتسبة لديه" الديه

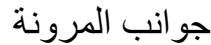

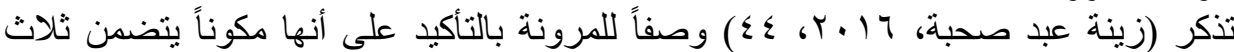

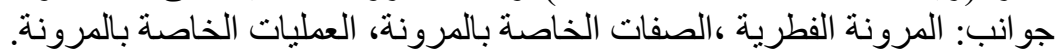
العوامل المؤثرة في المرونة المرنة

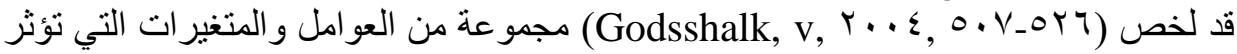

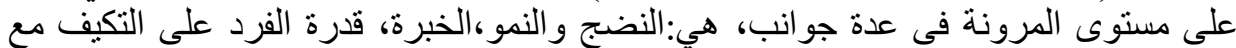

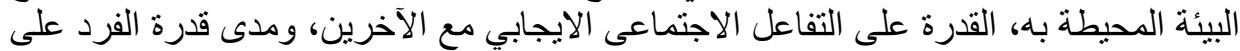

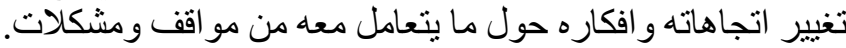
طرق بناء المرونة

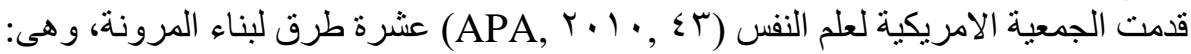

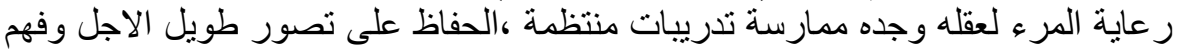

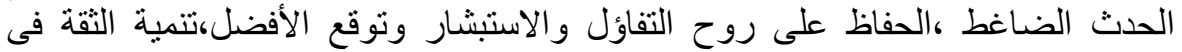

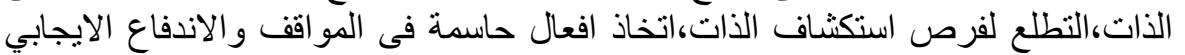

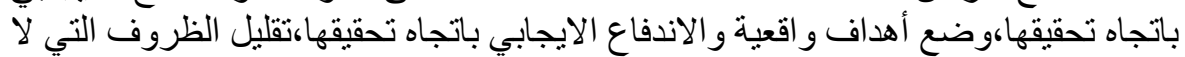

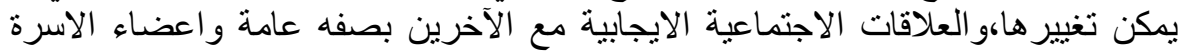
و الاصدقاء بصفة خاصة.

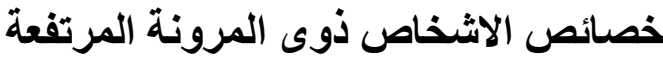

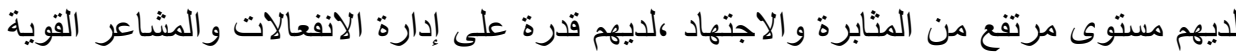

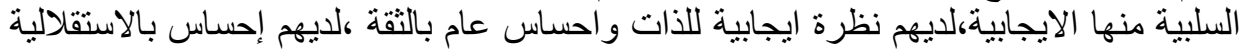

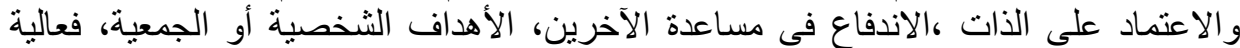

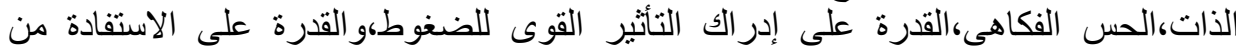

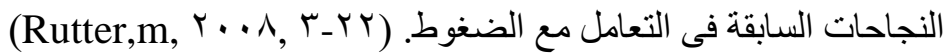

\section{دراسات تناولت المرونة و المرحلة الثانوية}

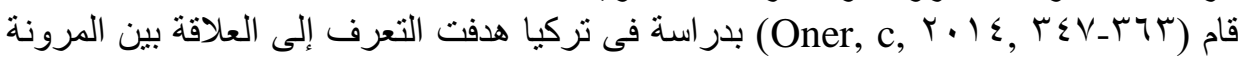

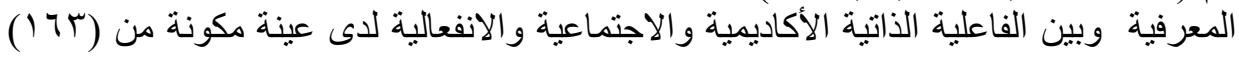

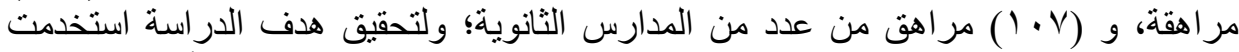

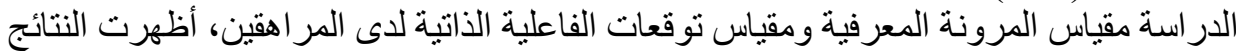

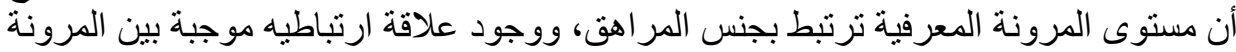

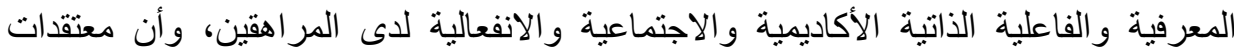

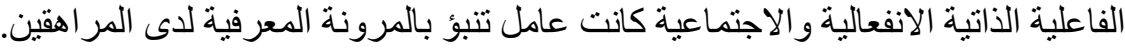

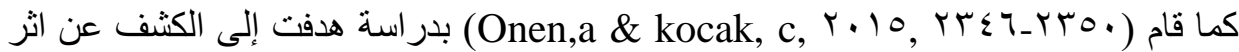

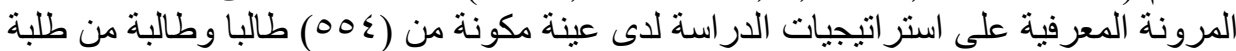

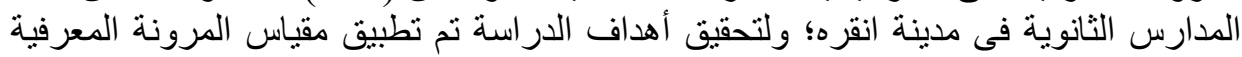


المعد من قبل (Bilgin) وكذلك مقياس استر اتيجيات الدراسة من قبل (Ozturk, k \& cetin).

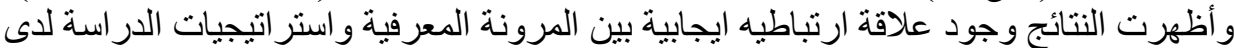
طلبة المدارس الثنانوية.

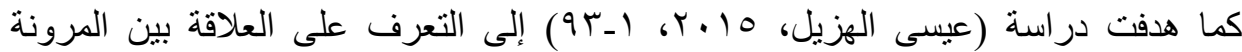

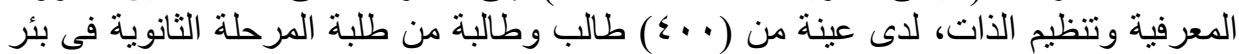

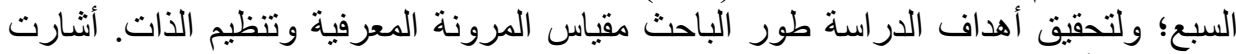

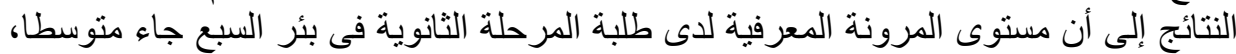

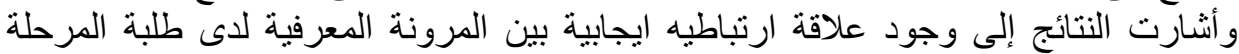

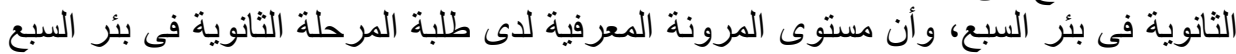

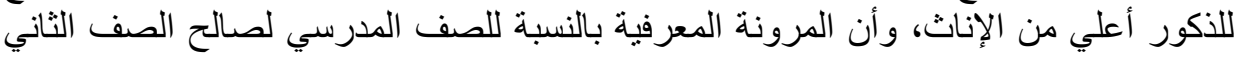

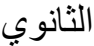

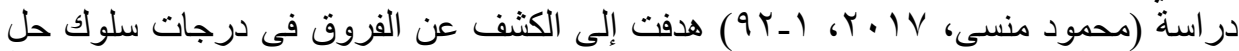

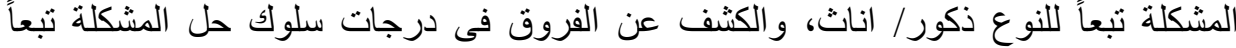

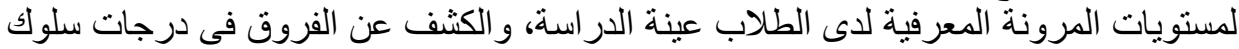

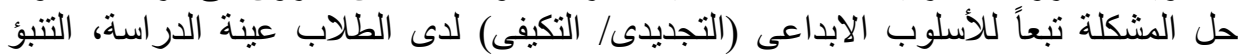

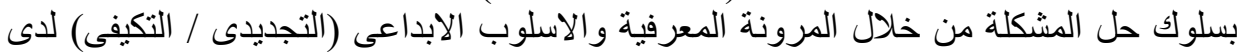

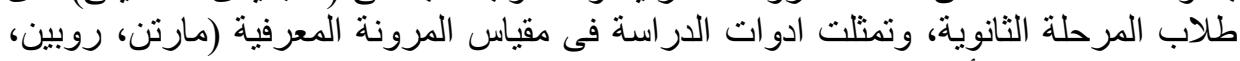

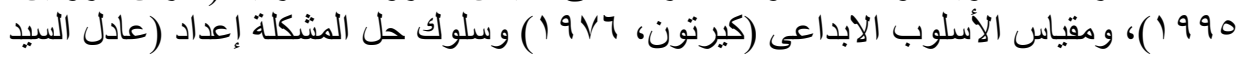

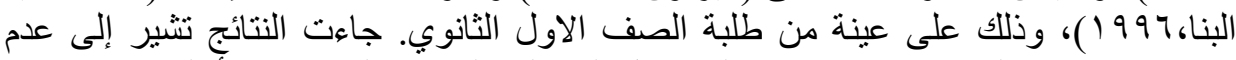

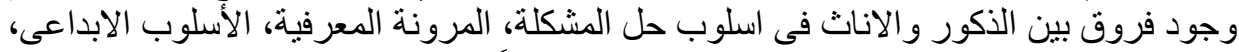

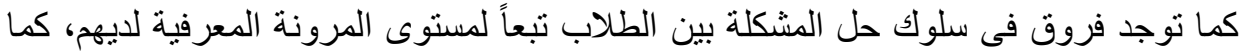

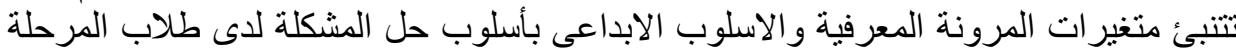

ا ـ الاطلاع على الأطر النظرية والدراسات و البحوث المتعلقة بمتغير ات البحث (الكفاءة

\section{إجراعات البحث}

الذاتية و المرونة).

r- ـ إعداد أدو ات البحثث و التي تتضمن مقياس الكفاءة الذاتية، ومقياس المرونة.

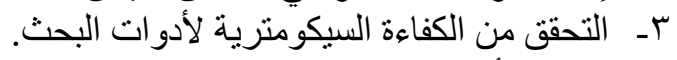

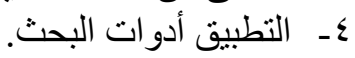

هـ ـ اختيار الأساليب الإحصائية الئناسبة للبحث.

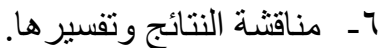

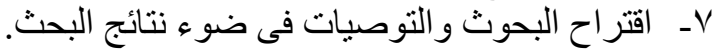




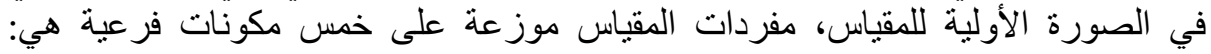

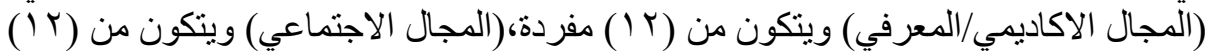

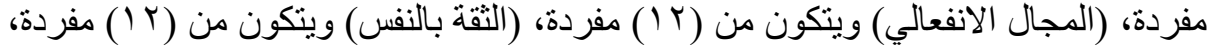

$$
\text { (تحمل المسئولية) ويتكون من (r) (Y) مفردة. }
$$

1ـ ـ الخصائص السيكومترية لمقياس الكفاءة الذاتية

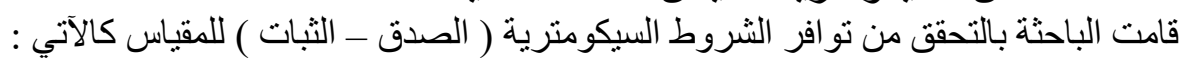
أو لا : ثبات المقياس: تم حساب ثبات المقياس بطريقة: معامل ألفا كرونباخ والتجزئئة النصفية

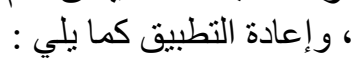

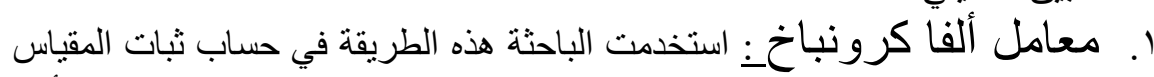

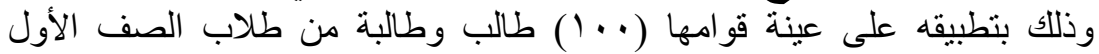

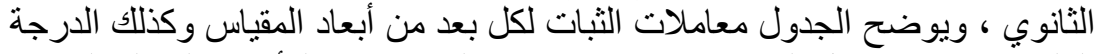

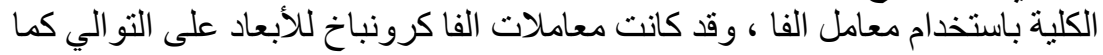

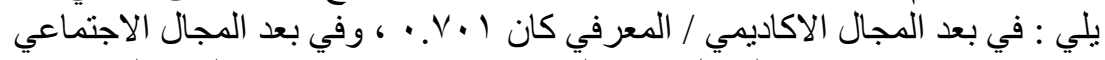

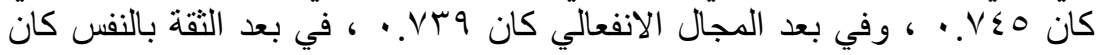

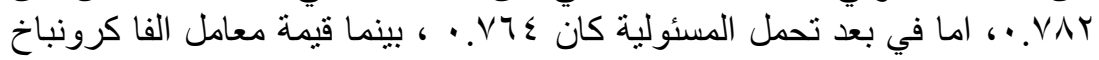

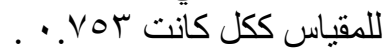
جدول (1 ) قيم معامل الثبات لكل بعد من أبعاد مقياس الكفاءة الذاتية وللمقياس ككل

\begin{tabular}{|c|c|c|}
\hline معامل ألفا كرونباخ & عدل العبارات & الأبعاد \\
\hline$\cdot . \mathrm{v} \cdot 1$ & $\overline{T Y}$ & المجال الاكاديمي / المعرفي \\
\hline$\because V \leq 0$ & IT & المجال الاجتماعي \\
\hline$\cdot . \vee r 9$ & IT & المجال الانفعالي \\
\hline •VAY & $\overline{T r}$ & الثقة بالنفس \\
\hline$\cdot . V 7 \varepsilon$ & IT & تحمل المسئولية \\
\hline . VOT & 7. & المقياس ككل \\
\hline
\end{tabular}




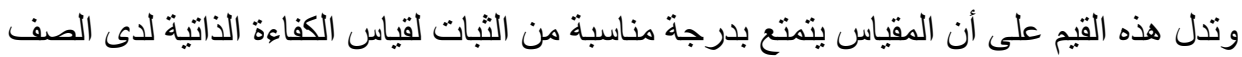

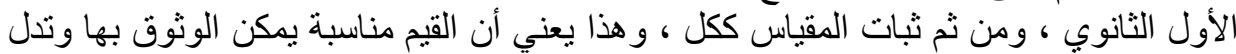

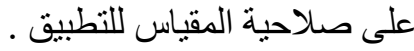

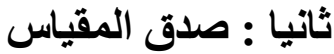
اعثمدت الباحثة في هذا البانيا البحث على صدق المحكمين للتأكيد على صدف المحتوى ، وكذلك

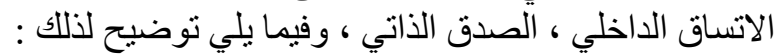

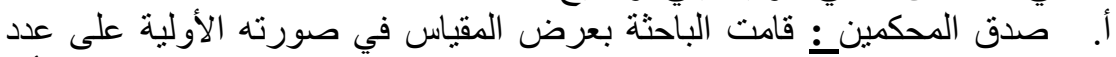

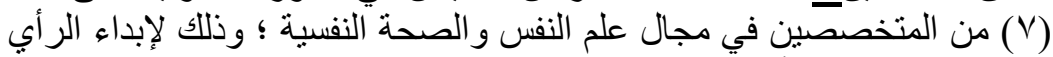

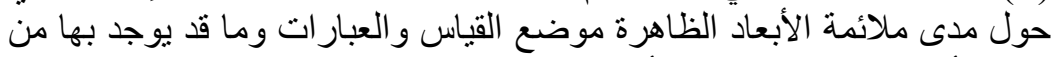

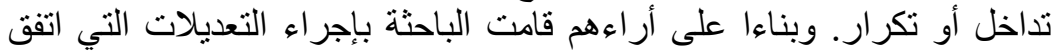

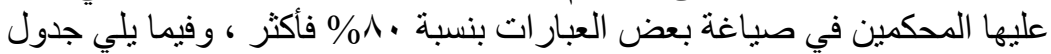

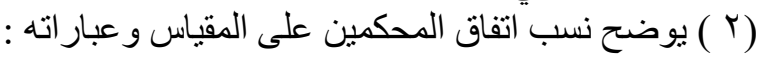

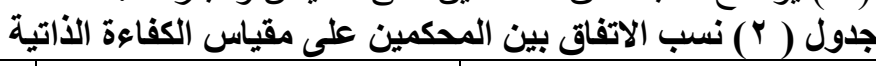

\begin{tabular}{|c|c|c|c|c|}
\hline \multirow{2}{*}{ نسبة الاتفاق } & \multicolumn{2}{|c|}{ الاتفاق بين المحكمين } & \multirow{2}{*}{ أبعاد المقياس } & \multirow{2}{*}{ ? } \\
\hline & غير موافق & موافق & & \\
\hline$\% 1 \cdots$ & . & $V$ & المجال الأكاديمي / المعرفي & 1 \\
\hline$\% 1 \ldots$ & . & $\bar{v}$ & المجال الاجتماعي & $T$ \\
\hline$\% 1 \ldots$ & $\cdot$ & V & المجال الانفعالي & $r$ \\
\hline$\% 1 \ldots$ & . & $\bar{v}$ & الثقة بالنفس & $\varepsilon$ \\
\hline$\% 1 \ldots$ & - & $\mathrm{V}$ & تحمل المسئولية & 0 \\
\hline$\% 1 \ldots$ & • & ro & & \\
\hline
\end{tabular}

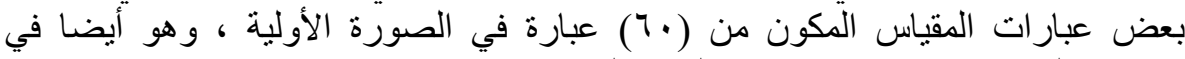

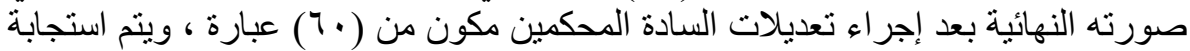

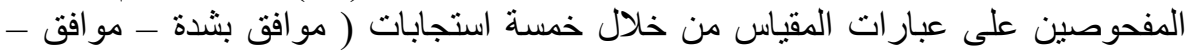

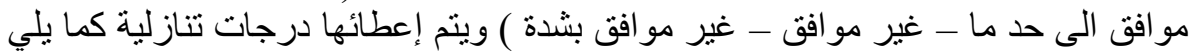

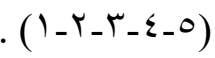

\section{مقياس المرونة (إعداد الباحثة)}

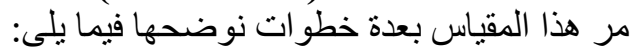

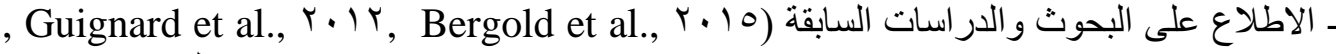

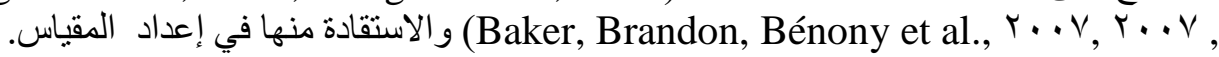

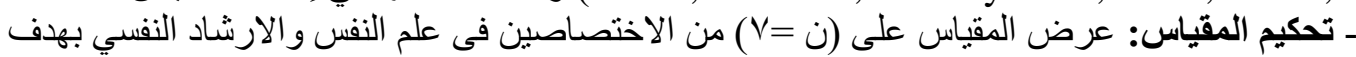

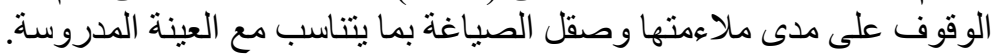

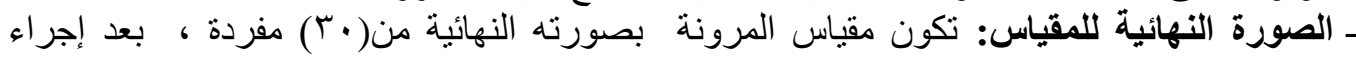
التعديلات في صياغة بعض العبار ات وإضافة التعريف الإجرائي علي المكون الفرعي في الصورة 
الأولية للمقياس، مفردات المقياس موزعة على ثلاثة مكونات فرعية هي: (التواصل الاجتماعي)

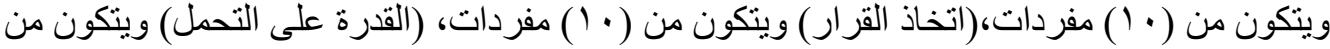

\section{1ـ الخصائص السيكومترية لمقياس المرونة}

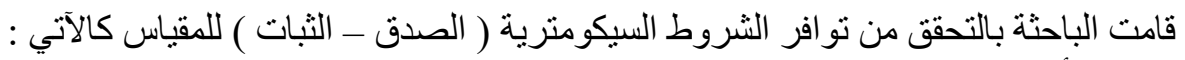

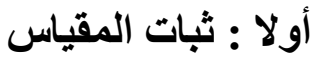

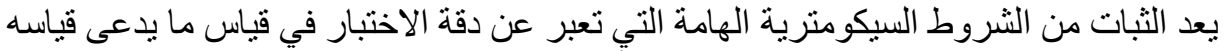
، وقد تم حساب ثبات المقياس بطريقة: معامل ألفا كرونباخ و التجزئة النصفية ، و وإعادة التطبيق

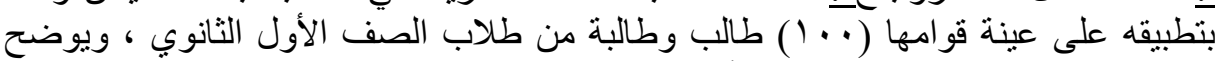

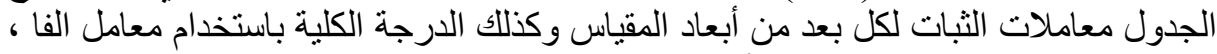

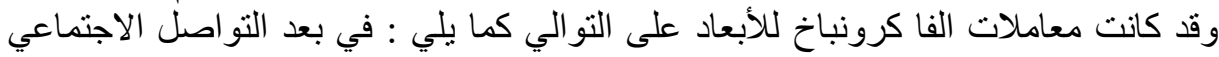

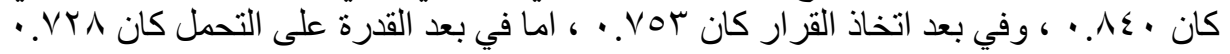

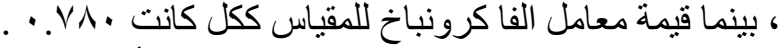

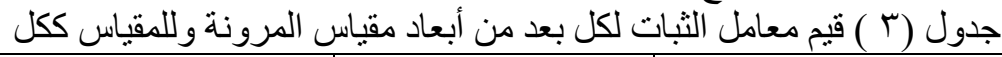

\begin{tabular}{|c|c|c|}
\hline معامل الفا كرونباخ & عدد العبارات & الأبعاد \\
\hline$\cdot \wedge \varepsilon$. & 1. & التواصل الاجتماعي \\
\hline. VOr & 1. & اتخاذ القرار \\
\hline.$V Y \wedge$ & 1. & القرة على التحمل \\
\hline$\because \vee \wedge$. & $r$. & المقياس ككل \\
\hline
\end{tabular}

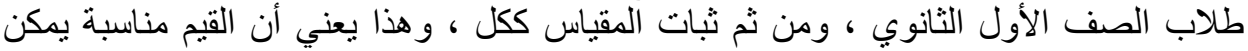

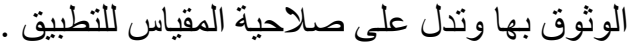

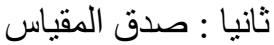

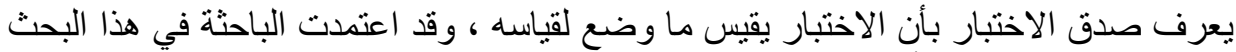

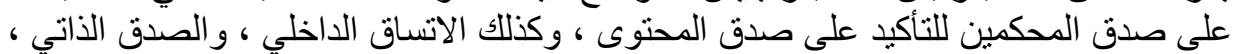

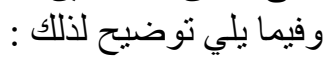

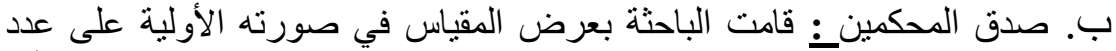

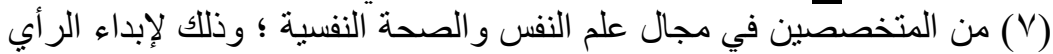

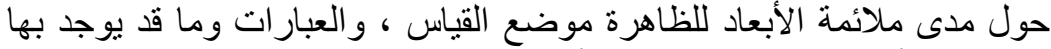

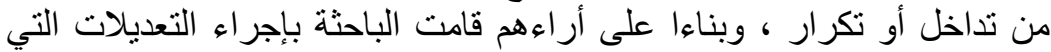

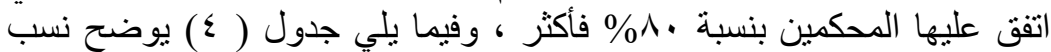
اتفاق المحكمين على المقياس و عبار اته : 


\begin{tabular}{|c|c|c|c|c|}
\hline \multicolumn{5}{|c|}{ جدول ( ؟) نسب الاتفاق بين المحكمين على مقياس المرونة } \\
\hline \multirow{2}{*}{ نسبة الاتفاق } & \multicolumn{2}{|c|}{ الاتفاق بين المحكمين } & \multirow{2}{*}{ أبعاد المقياس } & \multirow{2}{*}{ ? } \\
\hline & غير موافق & 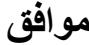 & & \\
\hline$\% 1 \ldots$ & . & $\mathrm{v}$ & التو اصل الاجتماعي & 1 \\
\hline$\% 1 \ldots$ & . & $\mathrm{v}$ & اتخاذ القر ار & $r$ \\
\hline$\% 1 \ldots$ & . & $\mathrm{v}$ & القدرة على التحمل & $r$ \\
\hline$\% 1 \ldots$ & . & YI & & \\
\hline
\end{tabular}

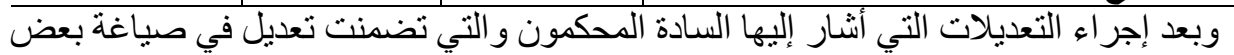

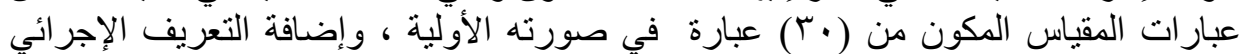

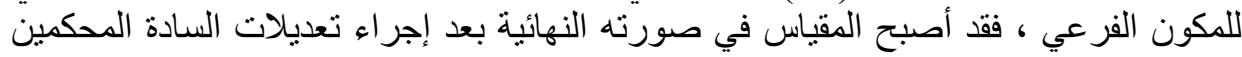

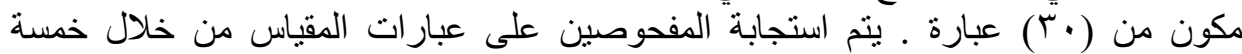

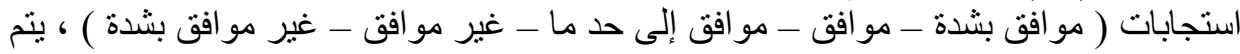

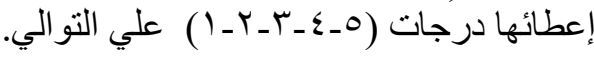

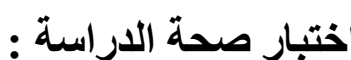

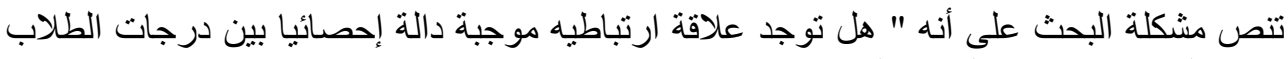

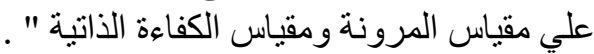

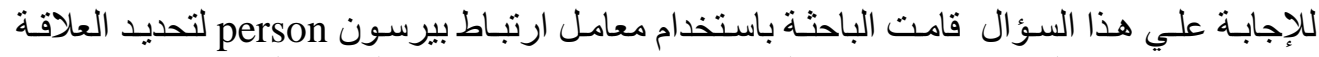

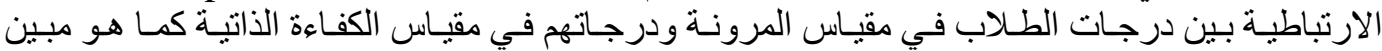

$$
\text { جدول (0) }
$$

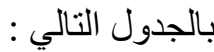

معامل الارتباط بين درجات الطالبات على مقياس المرونة ومقياس الكفاءة الذاتية

\begin{tabular}{|c|c|c|c|c|}
\hline نوع الارتباط & مستوى الدلالة & قيمة معامل الارتباط (ر) & أطراف العلاقة & العدد \\
\hline طردي موجب & $\cdot \cdot 1$ & .97 & المرونة × الكفاءة الذاتية & 0. \\
\hline
\end{tabular}

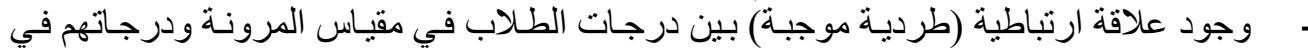

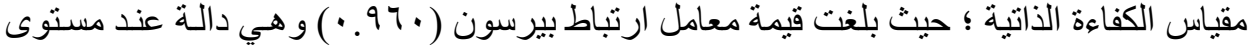




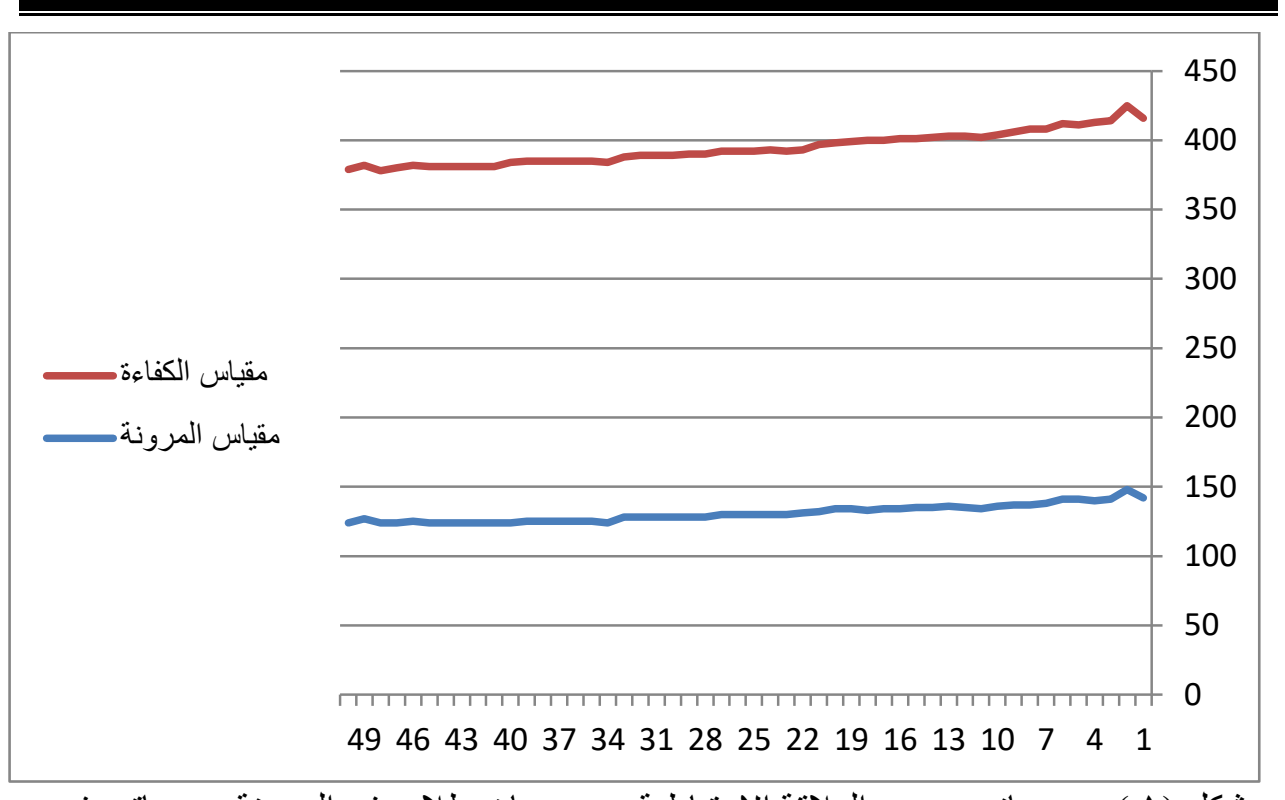

شكل ( 1 ) رسم بياني يوضح العلاقة الارتباطية بين درجات طلاب في المرونة ودرجاتهم في لكاني الكفاءة الذاتية

- ويمكن تفسير نتائج الدر اسـة فى ضوء الاطر النظريـة ونتائج الدراسـات السـابقة، وذلك على

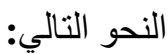

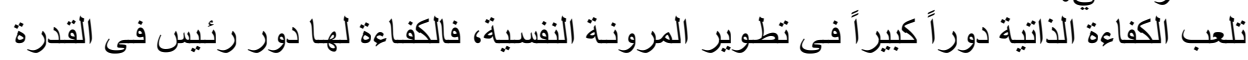

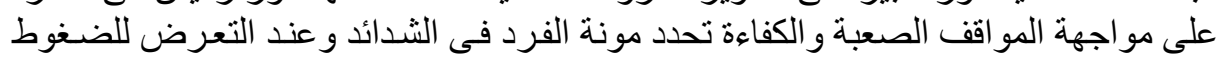

والاكتئاب.

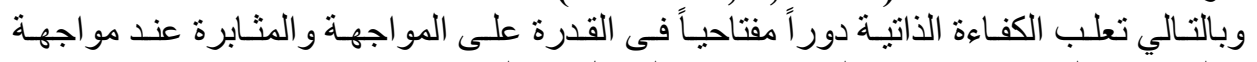

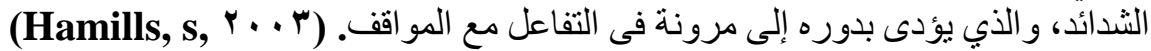

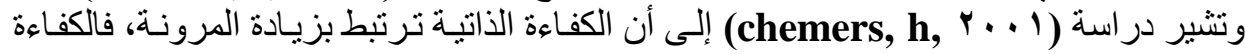

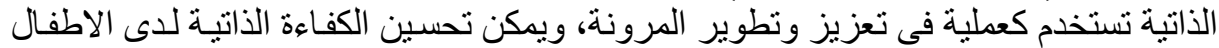

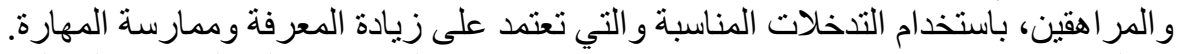

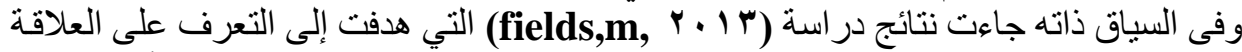

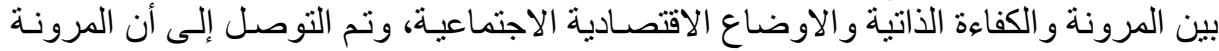

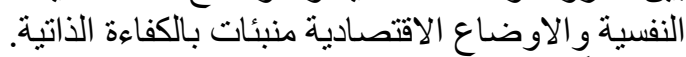

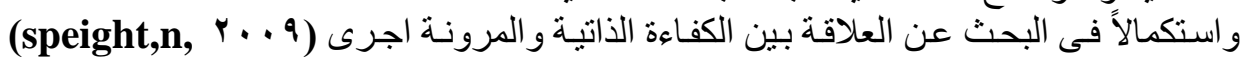

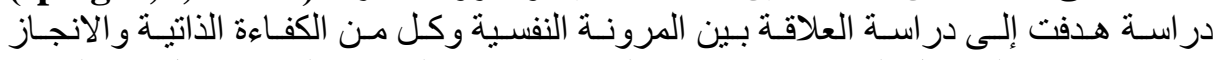

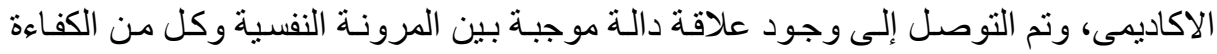

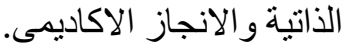




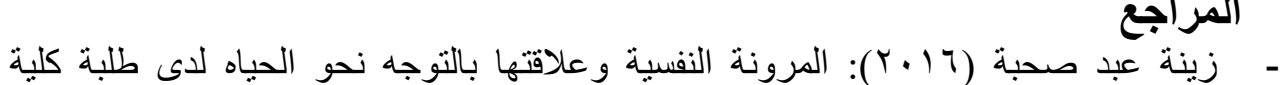

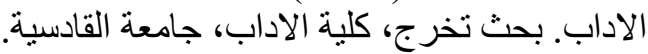

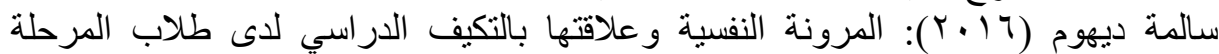

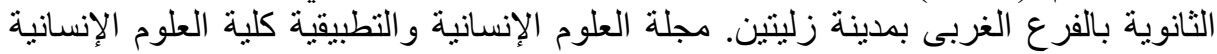

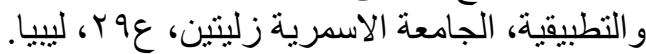

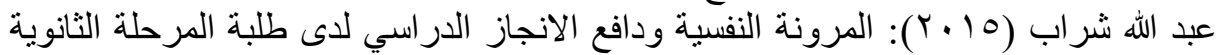

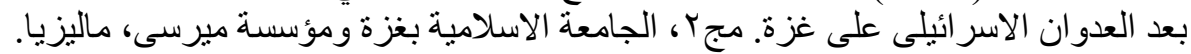

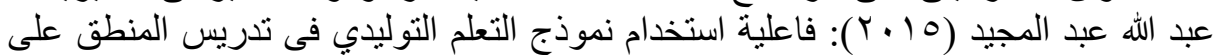

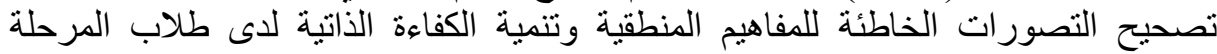

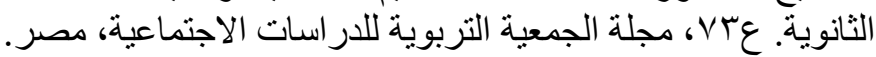

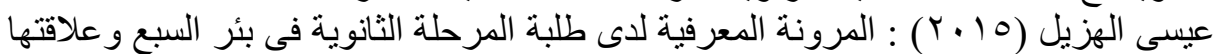

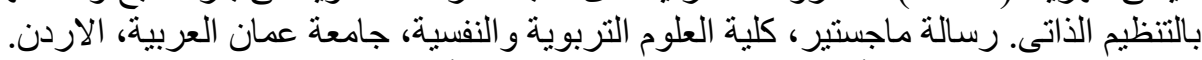

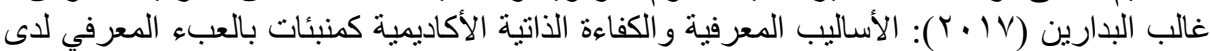

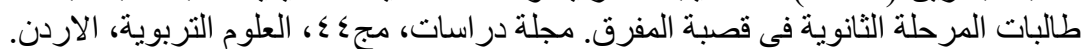

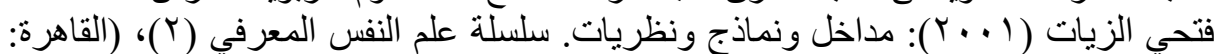

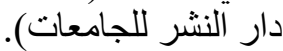

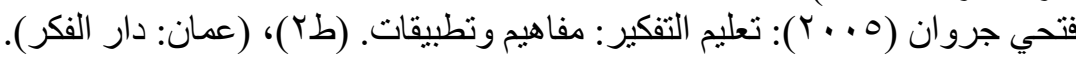

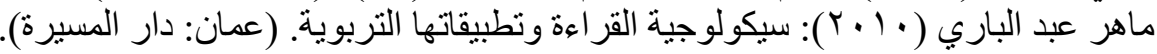

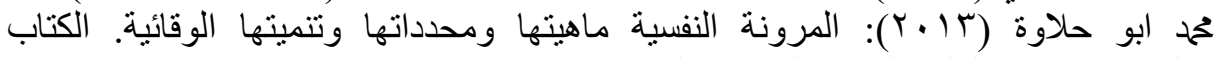
الإلكتروني شبكة العلوم النفسية العربية، العدد (Y) (Y). (Y).

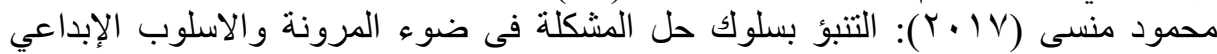
(التجديدي/ التكيفي) لاى طلاب المرحلة الثانوية. رسالة ماجستير، كلية التربية، جامعة الألة

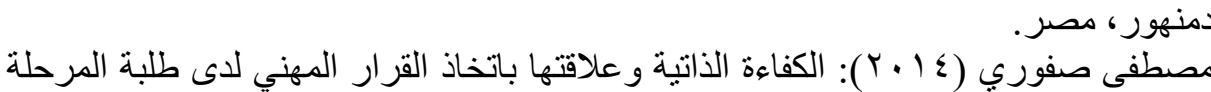

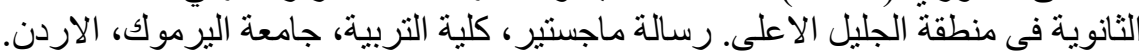

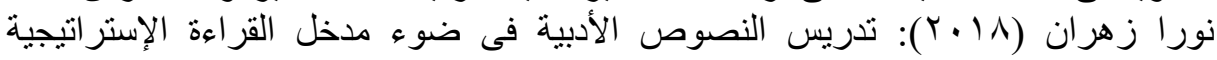

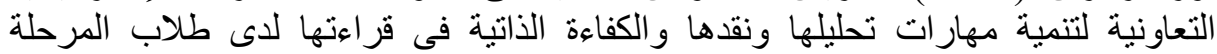

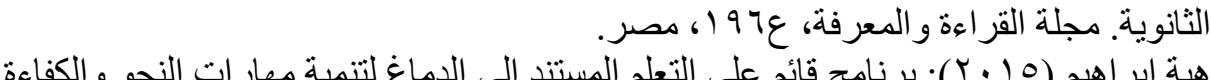

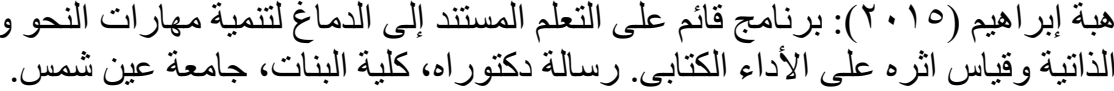

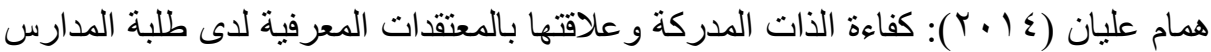

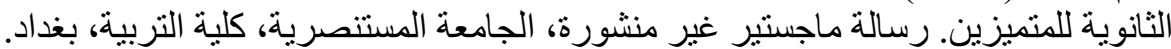

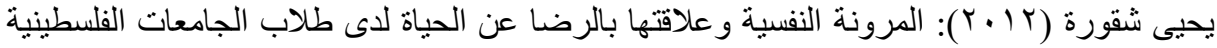

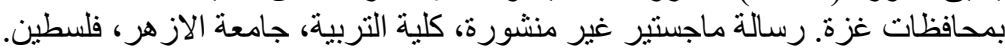
- Ahern , n ( $(\cdots \wedge)$ : Resilience and coping strategies in adolescents. Paediatric nursing, $r \cdot(l \cdot)$. 


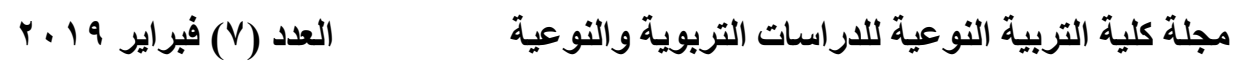

-American psychological association (APA) $(\uparrow \ldots 9)$ : The road to resilience. $V^{\bullet}$ •, first street, NE, Washington, DC.

-American psychological association (APA) $(r \cdot 1 \cdot)$ : Resilience and strength in black children and adolescents, avirision for optmal development.

-Bandura , a ( (99У): Self efficacy: the exercise of control. New York, W.H. freeman.

-Bandura, a $(r \cdots r)$ : Role of affective self regulatory efficacy in diverse spheres of psychological functioning child development, $\vee \leqslant$.

- Brookby S. ( $Y \cdots \varepsilon)$ : Academic self - efficacy and social self concept of mathematically Gifted high school student: in summer residential program. . (ed), psychology department, capella University.

- Bergold S, Wirthwein L, Rost DH, Steinmayr R.( $\left.\left(r^{\prime}\right)^{0}\right)$ : Are gifted adolescents more satisfied with their lives than their non-gifted peers? Frontiers in Psychology; 7: 17 r r.

- Bénony H, Van Der Elst D, Chahraoui K, Bénony C, Marnier JP. $(Y \cdots \vee)$ : Link between depression and academic self-esteem in gifted children. Encephale; $r \mathrm{r}(1): 11-r \cdot$ •

-Bong, $\mathrm{m}(\boldsymbol{Y} \cdot \mathrm{M})$ : Between and within domain relations of academic motivation among middle and high school students: self efficacy, task value, and achievement goals. South Carolina, dept of educational psychology, Columbia, sc, us.

- Castejón JL, Gilar R, Veas A, Miñano P.(Y• 1 ฯ): Differences in Learning Strategies, Goal Orientations, and Self-Concept between Overachieving, Normal-Achieving, and Underachieving Secondary Students. Frontiers in Psychology; $\vee: 1 \leqslant r \wedge$.

-Chemers, $h(Y \cdot$,$) : Academic self efficacy and first year college students per-$ formance and adjustment. Journal of educational psychology, 9 r.

- Cynthia A,edins. ( $(\ldots 9)$ : Self - efficacy and self - Esteem in Gifted and non Gifted in the elementary schools system .(ed), psychology department, Missouri-Kansas City University.

-Dennis , $\mathrm{j} \&$ vander, $\mathrm{j}(\mathrm{r} \cdot \mathrm{l} \cdot)$ : The cognitive flexibility inventory: instrument development and estimates of reliability and validity. Cognther res, $r \leqslant$.

-Fields, m $(\uparrow \cdot 1 r)$ : Resilience, socioeconomic status, and career decision self efficacy in rural Appalachian adolescents, $\mathrm{PhD}$, waiden university. 


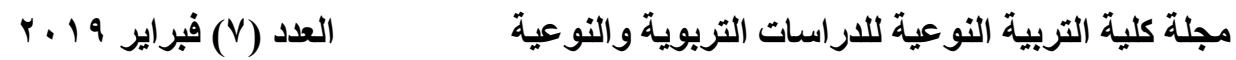

- Godsshalk, v ( $\uparrow, \ldots \varepsilon)$ : The role of learning tasks on attitude change using cognitive flexibility hypertext system. The journal of the learning sciences, 1 r.

- Guignard JH, Jacquet AY, Lubart TI. $(Y \cdot M)$ ): Perfectionism and Anxiety: A Paradox in Intellectual Giftedness? Rustichini A, ed. PLoS ONE ;

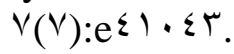

-Hamills , s ( $(\ldots r)$ : Resilience and self efficacy: the importance of efficacy beliefs and coping mechanisms in resilienent adolescents. Colgate university journal of the sciences, ro.

- Kalena, L. PETERSON, M.A. $(r \cdot 1 \leqslant)$ : Gifted African American: the Relationship Between self efficacy, familial support Educational attainment. Income, and life satisfaction a dissertation submitted to the faculty of the adler school of professional psychology.,(ed), professional psychology, the faculty of the adler school.

-Margolis, $h$ \& Mccabe, $p(r \ldots \tau)$ : Improving self efficacy and motivation: what to do, what to say. Intervation in school and clinic, $\varepsilon)(\varepsilon)$.

-Onen, a \& kocak, c $(\uparrow \cdot 10)$ : The effect of cognitive flexibility on higher school students study strategies. Social and behavioral sciences, 191.

-Oner, c $(r+1 \leqslant)$ : The relation between cognitive flexibility and academic. Social and emotional self efficacy beliefs among adolscents. Education \& science, $r q(\mid \vee\urcorner)$.

-Reivich , $\mathrm{k}(\boldsymbol{r} \mathrm{l} \cdot)$ :Building resilience in youth.The pen resiliency program, national association of school psychologists, $r \wedge(\top)$.

-Rose , a $(r \cdot 11)$ : Restorative environments influence on cognitive flexibility in developing adults. Thesis master of science, the university of Utah.

-Rutter , $m(Y \cdots \wedge)$ : Developing concepts in developmental psychopathology in J.J. hudziak (ed). Developmental psychopathology in fluencies. American psychiatric publishing.

-Sharm , $d(r \cdots v)$ : Revisiting an earn Germany from the perspective of adolescents in mother headed single parent families. International journal of psychology, $\leqslant r(1)$.

-Speight, $n(r \cdots 9)$ : The relationship between self efficacy, resilience and academic achievement among African American urban adolescent students, Ph.D. howard university.

-Wang, m ( (99v): Fostering educational resilience in inner-city schools. Office of educational research and improvement (ed), Washington. 